\section{Avaliação da cobertura assistencial das equipes de saúde da família às pessoas que faleceram por doenças cerebrovasculares em Maringá, Paraná, Brasil}

\author{
Evaluation of coverage by family health teams for \\ fatal stroke victims in Maringá, Paraná State, Brazil
}

\author{
1 Secretaria Municipal da \\ Saúde, Maringá, Brasil. \\ 2 Centro de Ciências \\ Aplicadas e da Saúde, \\ Pontifícia Universidade \\ Católica do Paraná, Maringá, \\ Brasil. \\ 3 Departamento de \\ Enfermagem, Universidade \\ Estadual de Maringá, \\ Maringá, Brasil. \\ 4 Departamento de Saúde \\ Coletiva, Universidade \\ Estadual de Londrina \\ Londrina, Brasil. \\ Correspondência \\ E. K. K. Baptista \\ Secretaria Municipal da \\ Saúde. \\ Av. Prudente de Moraes 885 \\ zona 7, Maringá, $P R$ \\ 87020-010, Brasil. \\ ebaptista@maringa.pr.gov.br
}

\begin{abstract}
The work process in the Brazilian Family Health Program (FHP) allows teams to establish bonds, accept families, and take responsibility for the registered population and identify groups at risk. Monitoring allows problem surveying and evaluation of specific measures. Considering that a key objective of controlling arterial hypertension is to reduce stroke mortality, this study aimed to identify the coverage, follow-up, and quality of care for individuals in areas with the FHP who died of stroke, based on data from the family registries (Form A), follow-up (Form $B)$, and patient records in primary health care units. The results showed low coverage, with $33.3 \%$ of registration on Form A, follow-up of $4.9 \%$ of deaths, and absence of any annotation on the care provided in most patient records (52.4\%). Analysis of care in the group confirmed the fragmentation and disconnection between the different information systems. It is essential to encourage the use of available data and improve their recording in order to institutionalize evaluation practice and work organization from a health surveillance perspective.
\end{abstract}

Cardiovascular Diseases; Hypertension; Information Systems; Family Health Program
Eliane Katsume Kikumoto Baptista 1,2

Sonia Silva Marcon 3

Regina Kazue Tanno de Souza 4

\section{Introdução}

As modificações na composição populacional, com o aumento da população acima de 60 anos, exigem olhares diferenciados sobre o padrão de morbi-mortalidade, agora com peso mais significativo das doenças crônico-degenerativas 1 . São atribuídas a essas doenças a responsabilidade por, aproximadamente, $50 \%$ das mortes, e é fato que os principais fatores de risco para a sua ocorrência são a obesidade, alto nível de colesterol, hipertensão arterial, tabagismo e alcoolismo ${ }^{2}$.

A hipertensão arterial é importante fator de risco para a ocorrência de agravos cardiovasculares que se constituem nas principais causas de óbito e hospitalizações 1,2,3. O acidente vascular encefálico é a primeira causa de incapacidades em adultos nos países industrializados 4 . Estimativas indicam que $40 \%$ dos acidentes vasculares encefálicos que incidem sobre os pacientes hipertensos, com o tratamento adequado, poderiam ser evitados 1,3.

Ademais, a hipertensão arterial é um dos problemas de saúde mais prevalentes. Calcula-se a existência de 600 milhões de hipertensos em todo o mundo ${ }^{2}$. No Brasil, a prevalência da hipertensão arterial varia de $40 \%$ a $50 \%$ da população acima de 40 anos 1 . A priorização do cuidado a esse segmento populacional é orientada pela Norma Operacional da Assistência à Saúde/SUS (NOAS-SUS 01/2001) 5, que estabelece, como responsabilidade dos municípios, as ações estra- 
tégicas em áreas prioritárias de saúde, entre elas, o controle da hipertensão arterial.

A implantação da estratégia de saúde da família exige visão ampliada do conceito de saúde ao introduzir concepções como de vigilância, integralidade, planejamento local, gestão colegiada e reorientação do trabalho na lógica programática, com ênfase nos grupos populacionais em situação de risco. Nessa abordagem, os sistemas de informação em saúde (SIS) têm papel relevante na construção de instrumentos que promovam o monitoramento e avaliação de metas, inclusive as pactuadas entre as esferas de governo 6 .

Considerando-se que uma dessas metas é a redução da mortalidade por doenças cerebrovasculares, por meio do controle da hipertensão arterial, este estudo tem por objetivo identificar a cobertura da assistência prestada pelas equipes do Programa Saúde da Família (PSF) das unidades básicas de saúde de Maringá, Paraná, às pessoas falecidas por doenças cerebrovasculares, a partir da freqüência dos registros nas diferentes fontes e sistemas de informação.

\section{Metodologia}

O estudo foi realizado em Maringá, município da região noroeste do Estado do Paraná, Brasil, com, aproximadamente, 400 mil habitantes, onde $84 \%$ da população é coberta pelo PSF. Foram analisados os óbitos de residentes em áreas cobertas pelo PSF, ocorridos em 2004, no município. Foram selecionados os casos cuja causa básica foi classificada, segundo a Classificação Estatística Internacional de Doenças e Problemas Relacionados à Saúde, 10a Revisão (CID-10) 7 e a parametrização do Sistema de Pactuação de Indicadores de Atenção Básica (SISPACTO) 6, como decorrente de doenças cerebrovasculares, acrescidos dos óbitos por outras causas com registro de doenças cerebrovasculares em qualquer parte da Declaração de Óbito (DO)

A cobertura e o acompanhamento pelas equipes de saúde da família foram identificados nas fichas de cadastro familiar (Ficha A) e de acompanhamento individual (Ficha B) do Sistema de Informação da Atenção Básica (SIAB), nos cadastros do Sistema de Acompanhamento de Hipertensos e Diabéticos (HIPERDIA) e nos prontuários. Os dados foram digitados no programa Excel (Microsoft Corp., Estados Unidos) e tabulados no programa TabWin (Ministério da Saúde, Brasil), adaptado para as variáveis selecionadas.

A pesquisa foi aprovada pelo Comitê de Ética em Pesquisa com Seres Humanos da Universidade Estadual de Maringá (parecer no. 093/2005).

\section{Resultados}

No ano de 2004, ocorreram, em Maringá, 1.582 óbitos de residentes. Em 200 casos, havia registro de doenças cerebrovasculares na DO como causa básica $(71,5 \%)$ e causas conseqüenciais ou contribuintes (28,5\%). Desses, 91,5\% (183) residiam nas áreas de abrangência das 22 unidades básicas de saúde do município. A maioria foi no ambiente hospitalar (84\%), com predomínio do sexo masculino (56\%) e de maiores de 70 anos (69,5\%).

A consulta aos diferentes SIS revelou a baixa localização de registros (Tabela 1). Ademais, em $12 \%$, a Ficha A foi a única fonte de dados; a Ficha B, em 0,5\%; o cadastro no HIPERDIA, em 2,7\%; e o prontuário, em $18 \%$ dos casos.

Dos 61 casos registrados na Ficha A, constatou-se que a hipertensão arterial e o acidente vascular cerebral estavam anotados em 63,9\% das fichas. No HIPERDIA, a hipertensão arterial foi referida em 14 dos 15 cadastros localizados, e, nos prontuários, o diagnóstico da hipertensão arterial ou do acidente vascular cerebral estava presente em $49,4 \%$ e 44,9\%, respectivamente.

Entre os 89 prontuários estudados, constatou-se que, em 19,1\% dos casos, o primeiro atendimento, realizado nas unidades básicas de saúde de referência, ocorreu há menos de um ano da data do óbito, e o maior número, no período de 1 a 4 anos (43,8\%). Quanto ao local de arquivamento, $37,1 \%$ estavam junto aos arquivos de prontuários ativos das unidades básicas de saúde. Apenas, em 4,5\%, havia anotações da data da ocorrência e a causa do óbito, e, em $14,6 \%$, a data do óbito.

\section{Tabela 1}

Cobertura das fichas cadastrais dos sistemas de informação em saúde e do prontuário da rede básica de saúde de Maringá, Paraná, Brasil, 2004.

\begin{tabular}{lcccc}
\hline \multirow{2}{*}{ Fontes } & \multicolumn{4}{c}{ Registro de dados } \\
& \multicolumn{3}{c}{ Sim } & Não \\
& n & $\%$ & n & $\%$ \\
\hline Ficha A & 61 & 33,3 & 122 & 66,7 \\
Ficha B & 9 & 4,9 & 174 & 95,1 \\
HIPERDIA & 15 & 8,2 & 168 & 91,8 \\
Prontuário & 89 & 48,6 & 94 & 51,4 \\
Uma das fontes & 123 & 67,2 & 60 & 32,8 \\
Todas as fontes & 2 & 1,1 & 181 & 98,9 \\
\hline
\end{tabular}

HIPERDIA: Sistema de Acompanhamento de Hipertensos e Diabéticos.

Fonte: unidades básicas de saúde de Maringá 


\section{Discussão}

O perfil de óbitos observado é compatível ao da literatura, com maior proporção em pessoas em idades avançadas e no sexo masculino. Embora a grande parte das ocorrências refira-se à população idosa, a discussão atual é quantos óbitos poderiam ser evitados com o adequado controle dos fatores de risco 3,8 . No caso das doenças cerebrovasculares, o reconhecimento da hipertensão como um dos agravos prioritários no monitoramento da qualidade da atenção básica tem justificado a sua inclusão nas pactuações intergovernamentais desde a Norma Operacional Básica do SUS (NOB-SUS) de $1996{ }^{9}$ e nos SIS. Assim, a situação da atenção a esse grupo populacional pode apresentar-se como traçador da atenção prestada pelas equipes de saúde da família aos grupos prioritários. Pela ênfase dada pelo serviço às doenças cerebrovasculares e a magnitude do óbito como agravo à saúde, esperava-se que $100 \%$ desses eventos fossem registrados no SIS.

Todavia, a baixa cobertura do preenchimento das fichas indica que o processo de trabalho das equipes de saúde da família não é orientado pelo princípio da vigilância, pois toda família residente deveria ser cadastrada na ficha $\mathrm{A}$; as pessoas com hipertensão arterial referida, na Ficha B; e, entre esses, os acompanhados pela unidade básica de saúde, no HIPERDIA 10,11.

Para o PSF, entendido como uma estratégia de mudança das práticas sanitárias, a contextualização da família no território/campo de trabalho é imprescindível, pois aí que reside a possibilidade de os profissionais ampliarem os limites de atuação. A incorporação dos agentes comunitários de saúde na equipe foi para responder a essa demanda organizacional. As principais atividades desse profissional são o cadastro e acompanhamento das famílias e, para tal, deve ser programada, pelo menos, uma visita mensal para cada família. Pelo cenário descrito, é de se supor que a prática de supervisão do trabalho do agente comunitário de saúde e a apropriação dos dados pela equipe de saúde da família, para avaliação da melhoria da qualidade, não constituem atividade regular.

Quanto aos prontuários, considerados importantes documentos de amparo legal e institucional, embora tenham sido a fonte de dado com maior proporção de registro, os 48,6\% localizados estão muito aquém dos resultados da avaliação normativa 12 realizada no país, onde mais de $89 \%$ das equipes referiram a utilização do prontuário para registro das atividades. Acresce, como fragilidade do serviço, o fato de $37,1 \%$ dos prontuários integrarem o arquivo ativo das unidades básicas de saúde.

A baixa identificação e a insuficiente localização das informações nos diferentes SIS demonstram que o trabalho das equipes de saúde da família ainda se organiza sem planejamento local, monitoramento e avaliação. Ao considerar os registros encontrados, em relação ao atendimento ao usuário, verifica-se a insuficiência da assistência em seus aspectos quantitativos e qualitativos e sugere que a situação não se restringe às mortes estudadas.

O padrão de cobertura apresentado ratificou a fragmentação, o paralelismo e a desarticulação entre as áreas. É muito provável que a situação observada no município seja um dos reflexos das políticas na área da informação em saúde, que, ao longo dos anos, tem se criado um SIS para cada demanda específica, sem a preocupação em seguir padrões e processos integrados. De modo geral, as políticas locais de informação em saúde são incipientes e centralizadoras, implantadas e mantidas conforme as demandas e exigências do Ministério da Saúde. A maioria das experiências municipais demonstra essa situação, reflexo das políticas nacionais de informação em saúde, pois, na agenda nacional, predomina a discussão acerca da obrigatoriedade do preenchimento de cadastros, fichas e relatórios e a alimentação dos diferentes SIS, para alocação de recursos financeiros. Nesse sentido, iniciativas como o "aprimoramento e a integração dos sistemas de informação em saúde" 13, a partir da construção cooperativa e horizontal, a pactuação intergestores e divulgação das experiências municipais exitosas são dignas de destaque. É reconhecer a necessidade de viabilizar a adoção de práticas locais que organizem o processo de trabalho em consonância com as metas programadas e pactuadas entre os níveis de gestão visando ao enfrentamento efetivo das adversidades em saúde, contexto no qual o uso dos dados e a melhoria do seu registro são imprescindíveis. 


\section{Resumo}

O processo de trabalho no Programa Saúde da Família (PSF) permite às equipes estabelecer relações de vínculo, acolhimento e responsabilização pela população adscrita e identificar grupos populacionais em situação de risco. O monitoramento proporciona o levantamento de problemas e avaliação das ações executadas. Considerando-se que um dos propósitos do controle da hipertensão arterial é a redução da mortalidade por doenças cerebrovasculares, o objetivo deste trabalho foi identificar a cobertura, acompanhamento e qualidade da assistência prestada às pessoas residentes em áreas de atuação do PSF que faleceram por doenças cerebrovasculares, a partir da consulta aos cadastros familiares (Ficha A), acompanhamento (Ficha B) e prontuários nas unidades básicas de saúde. Os resultados revelaram baixa cobertura, com $33,3 \%$ de cadastramento na Ficha A, acompanhamento de 4,9\% dos óbitos e ausência de anotação de atendimento nos prontuários como situação predominante $(52,4 \%)$. A análise da assistência a esse grupo ratificou a fragmentação e desarticulação entre os diferentes sistemas de informação. Estimular o uso dos dados disponíveis e melhorar o seu registro são imprescindiveis para a institucionalização da prática avaliativa e organização do trabalho na ótica da vigilância à saúde.

Doenças Cardiovasculares; Hipertensão; Sistemas de Informação; Programa Saúde da Família

\section{Referências}

1. Ministério da Saúde/Organização Pan-Americana da Saúde. Avaliação do plano de reorganização da atenção à hipertensão arterial e ao diabetes mellitus no Brasil. Brasília: Ministério da Saúde; 2004.

2. Organização Pan-Americana da Saúde. Doenças crônico-degenerativas e obesidade: estratégia mundial sobre alimentação saudável, atividade física e saúde. Brasília: Organização Pan-Americana da Saúde; 2003.

3. Firmo JOA, Barreto SM, Lima-Costa MF. The Bambui Health and Aging Study (BHAS): factors associated with the treatment of hypertension in older adults in the community. Cad Saúde Pública 2003; 19:817-27.

\section{Colaboradores}

E. K. K. Baptista, S. S. Marcon e R. K. T. Souza participaram do planejamento da pesquisa, coleta e análise dos dados, elaboração e revisão do artigo.

\section{Agradecimentos}

À Fundação Araucária de Apoio ao Desenvolvimento Científico e Tecnológico do Paraná da Secretaria de Estado da Ciência, Tecnologia e Ensino Superior do Paraná, ao Ministério da Saúde e ao Conselho Nacional de Desenvolvimento Científico e Tecnológico.
4. Falcão IV, Carvalho EMF, Barreto KML, Lessa FJD, Leite VML. Acidente vascular cerebral precoce: implicações para adultos em idade produtiva atendidos pelo Sistema Único de Saúde. Rev Bras Saúde Matern Infant 2004; 4:95-102.

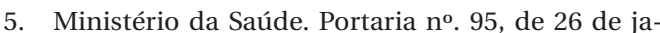
neiro de 2001. Norma Operacional da Assistência à Saúde/SUS. NOAS-SUS 01/2001. Diário Oficial da União 2001; 29 jan.

6. Ministério da Saúde. Portaria $n^{\circ}$. 2.394/GM, de 19 de dezembro de 2003. http://dtr2001.saude.gov. $\mathrm{br} / \mathrm{sas} /$ PORTARIAS/Port2003/GM/GM-2394.htm (acessado em 22/Dez/2003). 
7. Organização Mundial da Saúde. Classificação estatística internacional de doenças e problemas relacionados à saúde, 10a revisão. São Paulo: Centro Colaborador da OMS para a Classificação de Doenças em Português; 1995.

8. Ministério da Saúde. Portaria no ${ }^{\circ}$ 2.528/GM, de 19 de outubro de 2006. http://dtr2001.saude.gov. $\mathrm{br} / \mathrm{sas} /$ PORTARIAS/Port2006/GM/GM-2528.htm (acessado em 29/Jun/2007).

9. Ministério da Saúde. Norma Operacional Básica do SUS. NOB-SUS 01/96. Diário Oficial da União 1996; 6 nov.

10. Ministério da Saúde. Monitoramento da atenção básica de saúde: roteiros para ação e reflexão. Brasília: Ministério da Saúde; 2004.
11. Silva AS, Laprega, MR. Avaliação crítica do Sistema de Informação da Atenção Básica (SIAB) e de sua implantação na região de Ribeirão Preto, São Paulo, Brasil. Cad Saúde Pública 2005; 21:1821-8.

12. Ministério da Saúde. Avaliação normativa do Programa Saúde da Família: monitoramento da implantação e funcionamento das equipes de saúde da família: 2001-2002. Brasília: Ministério da Saúde; 2004.

13. Área de Informação e Informática em Saúde, Secretaria Executiva, Ministério da Saúde. Informação e informática no SUS: projetos, diretrizes e objetivos do Ministério da Saúde. http//labmodel. ensp/ iis2003 (acessado em 10/Out/2003).

Recebido em 14/Mar/2007

Versão final reapresentada em 10/Jul/2007

Aprovado em 05/Set/2007 